\title{
Self-monitoring Method for Improving Health-related Quality of Life: Data Acquisition, Monitoring, and Analysis of Vital Signs and Diet
}

\author{
Lina $\mathrm{Yu}^{1}\left(\right.$, Sha $\mathrm{Tao}^{2}\left(\right.$, Wanlin $\mathrm{Gao}^{2, *}(1)$ and Limin $\mathrm{Yu}^{3}(0)$ \\ ${ }^{1}$ Institute of Semiconductors, Chinese Academy of Sciences, Beijing 100083, China \\ ${ }^{2}$ College of Information and Electrical Engineering, China Agricultural University, Beijing 10094, China \\ ${ }^{3}$ College of Information Science and Engineering, Shandong Agriculture and Engineering University, Shandong 250100, China \\ * Corresponding author: Wanlin Gao (gaowlin@cau.edu.cn)
}

Manuscript Revised 26 April 2021; Accepted 26 April 2021; Published 27 April 2021

Academic Editor: Weiwei Cai (iD

Abstract: Vital signs are a series of clinical measurements and important to health-related quality of life. To establish a method for self-monitoring and management of vital signs and diet, a self-monitoring method (SMM) including wireless body area network and mobile technology was proposed in this paper. The study population comprised a total of 180 participants. Differences between measurements taken using the SMM and traditional instruments were analyzed with respect to accuracy and reproducibility. Participant measurements before and after intervention were used to evaluate the validity of the SMM. There was no statistically significant difference between our SMM and traditional instruments for measuring vital signs $(p>0.05)$. The relative standard deviation of the SMM $(0.38 \%)$ indicated good repeatability. These findings suggest that the SMM had a clear effect of promoting improvement in health habits, health condition monitoring, and disease prevention $(\mathbf{p}<0.05)$. Statistical analysis indicated that our SMM contributed to improve monitoring of vital signs and diet, and improved the health-related quality of life among study participants to a certain degree.

Index Terms: Vital signs, Self-monitoring, Wireless body area network, Mobile technology.

\section{Introduction}

Vital signs are a series of clinical measurements, including temperature (Temp), blood pressure (BP), pulse rate (PR), and blood oxygen saturation ( $\mathrm{SpO} 2)$, among others [1-3]. These indications are used to assess a person's physical health, possible disease states, and critical clinical conditions. In clinical practice, vital sign monitoring is an important activity carried out by medical professionals, to monitor patient progress and detect any irregularities [4-5]. In some cases, vital sign monitoring can assist in detecting health risks so that appropriate measures may be taken. Therefore, regular monitoring of vital signs can not only help with understanding the progression of disease, it can be used to promote improvement in the overall level of health [6]. Thus, vital sign monitoring provides a foundation for health care, including disease prevention and diagnosis [7].

With increasing health concerns among populations worldwide, the importance of understanding the role of vital signs and physical changes in daily life has grown. A balanced diet can supply daily necessary nutrition as well as assist in the prevention of many diseases. On the contrary, improper eating habits may cause health problems or worsen existing ones [810]. Consequently, there is a growing focus on improving physical conditioning by means of dietary control rather than by medical means. This might be reflected in the fact that 
dietary guidance is increasingly incorporated into treatment programs by physicians [11-12].

Accurate physiological information can be obtained using advanced medical equipment in a hospital setting. However, this approach costs a great deal of time and money and is a cumbersome method for tracking health. Traditional instruments, such as the thermometer and sphygmomanometer are inexpensive to use, but it is difficult to conduct comprehensive statistical analysis on the data acquired using such means. Regular monitoring of vital signs and dietary supervision in daily life is challenging without the help of medical professionals and medical devices [13]. Nevertheless, if, for example, individuals with hypertension or cardiovascular disease consume too much salt, their course of disease may be worsened [14-15] and could lead to a life-threatening situation without proper medical attention. In particular, older adults who are living alone need long-term, sustainable health care [16-17]. The question of how to implement the monitoring and analysis of vital signs and diet in a convenient, inexpensive, and consistent manner in real time has become the key to improving health-related quality of life (HRQoL).

It is widely accepted that the development of sensor technology and telemedicine systems has brought about many medical advances [18-21]. A number of remote monitoring devices have been used for vital sign measurement in medical settings. Such instruments provide medical services free from the limitations of time and location, and have had a positive role in clinical treatment of disease [13, 22-24]. However, a person's diet is affected by many elements such as region, culture, habits, preference, and even psychology [11, 25-27]. Therefore, a lack of monitoring tools has resulted in a situation where the status of dietary health among various populations remains largely unknown in the public health landscape. To date, there have been no effective methods created for measuring nutrient intake [28-31].

Given the above, as well as the importance of vital signs and diet in human health, this research proposes a self-monitoring method (SMM) for obtaining these two kinds of data. The SMM presented in this article can help to detect the warning signals of physical disease and an unhealthy diet, by obtaining data of vital signs and dietary nutrition. These data, together with a person's medical history, can be used to explore interactions between diet and disease. This process may provide a solution for improving HRQoL.

\section{Methods}

The SMM developed in this study is composed of a series of wearable self-monitoring devices (WSMD) and a health selfmanagement system (HSMS), as shown in Fig. 1. The principal function of the WSMD to collect physiological parameters through a series of low-power, noninvasive, and micro-sensor nodes based on WBAN (wireless body area network) technology [32-33]. The WSMD then sends these data to the
HSMS for storage and analysis using Bluetooth and $\mathrm{Wi}-\mathrm{Fi}$ wireless communication technologies [34-35]. In this study, the WSMD measured parameters including Temp, BP, PR, and SpO2. The YSI-400 temperature sensor (YSI Inc., Yellow Springs, OH, USA) was used, as well as the FGN-605PGSR pressure sensor (Fujikura (China) Co., Ltd., Shanghai, China) for BP signal acquisition. In addition, we obtained BP and $\mathrm{SpO} 2$ data by calculating different transmittance using fingertip pulse wave analysis. Using the WSMD, individuals can easily measure vital sign parameters at home at key time points and save the data on their mobile phones. Also, physicians can establish a personalized treatment program through observing changes in the patient's mobile data. HRQoL can be dramatically improved as a result, especially for patients with chronic diseases.

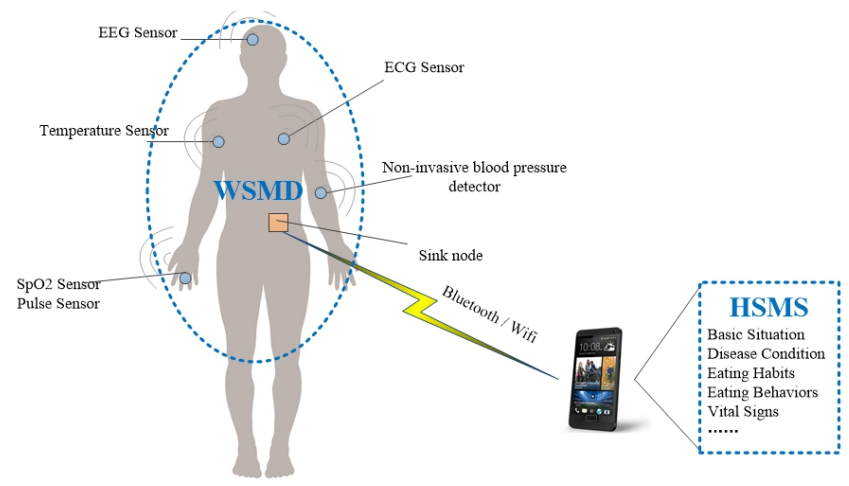

Fig.1. Design of the SMM.

The HSMS is a mobile phone application (app) that contains four modules. In the "Today" module, the intake of calories and nutrients, as well as data describing vital signs, are presented graphically. Vital sign statistics can be seen in more detail in the module "Vital Signs". In addition, users can add to and manage their daily dietary intake using the function entitled "Food". Furthermore, the user's height, weight, dietary habits, medical conditions, and other personalized information are recorded in the module "My". Several display screens from the HSMS are shown in Fig. 2.

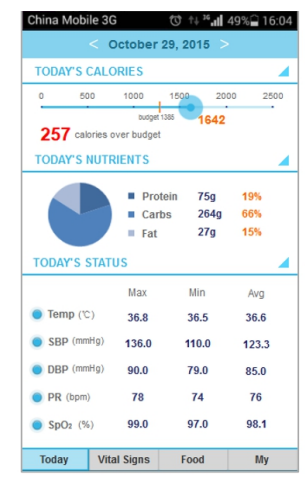

(1) Current situation

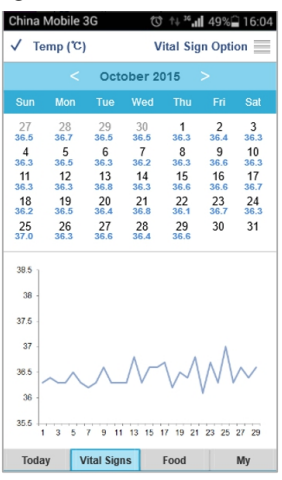

(2) Monthly data of vital signs

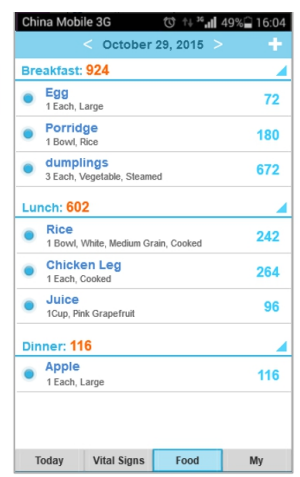

(3) Dietary record
Fig.2. Sample displays of the HSMS app.

The SMM self-monitoring process is illustrated in Fig. 3. With respect to self-monitoring of vital signs, first the WSMD receives physiological data using several sensors and then sends these to the HSMS. Next, the data are stored on the HSMS in a Vital Signs Database (VSDB) and compared with a Rules 
Database $(\mathrm{RDB})$ containing normal reference ranges. If outliers are present within the dataset, the "Vital Sign" module of the HSMS will respond by giving a warning. With regard to dietary self-monitoring, detailed dietary records are input by the user in the "Food" module. These are stored in a Diet Database (DDB) and translated in terms of nutrients by querying a Food-Nutrition Database (FNDB). Relationships are determined between reference data from a Dietary Recommendation Database (DRDB) and the user's personalized data entered into the "My" module and recorded in a Personal Basic Database (PBDB). Information collected by the WSMD and HSMS over a period of use can provide regular weekly, monthly, and annual data for analysis.

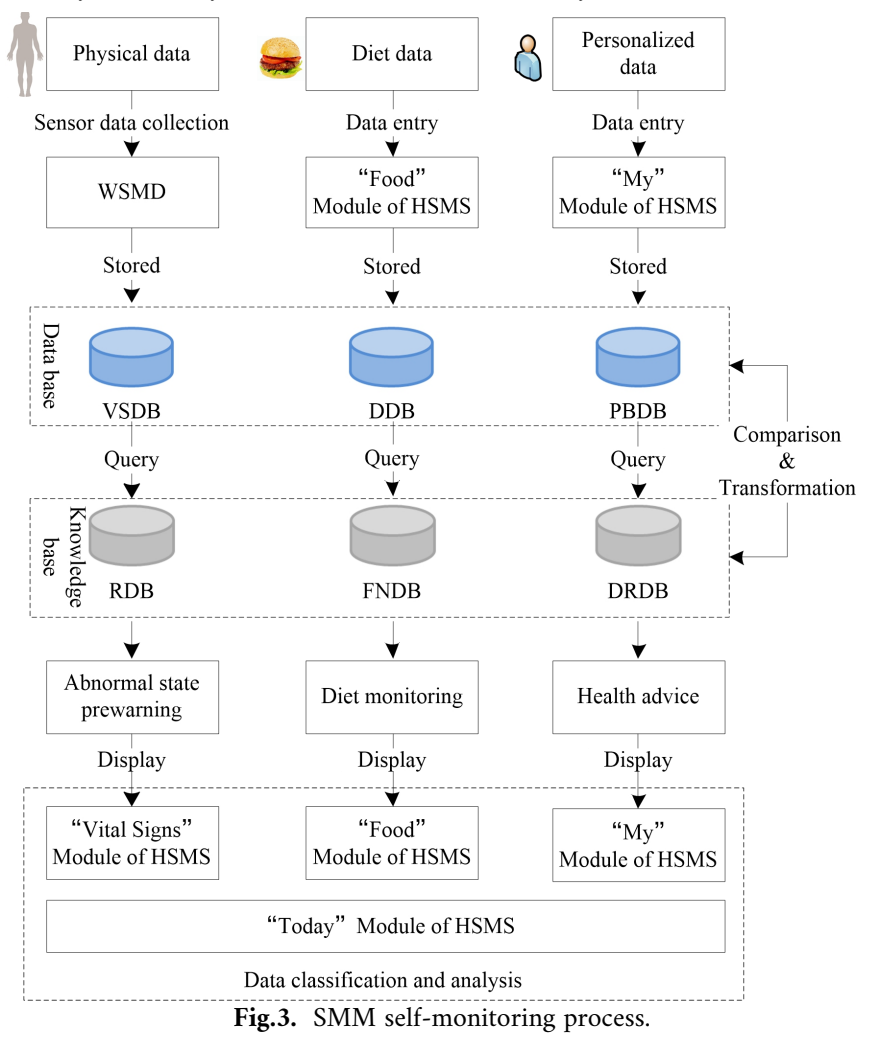

\section{Experiments}

Participants were recruited online. To facilitate communication during the intervention, recruitment was mainly focused in northern China, including the provinces of Beijing, Tianjin, Hebei, Shanxi and Inner Mongolia. The inclusion criteria were: (1) aged 18 years or above (2) owner of a smartphone with an Android or iOS operating system, and (3) willingness to complete the testing and questionnaires. The SMM is not intended only for those with a medical condition, so the presence of a disease was not a requirement.

Questionnaires were used to obtain participant characteristics such as sex, age, education level, and income. After receiving training, the HSMS was installed on each participant's smartphone. Each participant was given a WSMD and was expected to monitor their vital signs with it and keep a food log using the HSMS, according to their own schedule. Telephone follow-up was conducted after 3 months, to evaluate the effectiveness of the SMM.
To assess the accuracy and reproducibility of WSMD data measurement, a random sample of participants was analyzed. One participant was requested to perform normal daily activities and collect vital sign data, using both the WSMD and traditional instruments simultaneously. Two tests were performed. First, data acquisition was hourly between 8:00 and 22:00, with a time lag of 5-10 minutes to allow all parameters to return to their normal state. Vital signs were then continuously monitored by the WSMD every 2 minutes. After acquisition, statistical analysis of the data was carried out using IBM SPSS Version 19.0 software (IBM Corp., Armonk, NY, USA) and a t-test procedure. The level of statistical significance was set at 0.05 .

To study the effectiveness of the SMM, we established a basic questionnaire consisting of three questions. Q1: How often do you record your dietary intake? Q2: Do you choose food and cooking methods according to your health status? Q3: Have you ever observed physical changes after eating? For each question, participants had to choose one of the following responses: Never, Seldom, Often, Usually, or Always. Participants completed the questionnaire both before and after the intervention. The responses were compared and analyzed to measure the influence of the SMM. We applied the Wilcoxon signed-rank test for the comparative analysis. The difference and rank of responses were calculated using IBM SPSS software, and the significance level was set at 0.05 .

\section{Results}

\subsection{Participant characteristics}

The validation study sample comprised 180 volunteers. Participant age, sex distribution, educational level, and other features are summarized in Table 1 . A breakdown of the study sample showed the following: 84 (46.67\%) were men and 96 (53.33\%) women; the majority (75\%) were under age 50 years; half of them (50\%) had completed tertiary education; and $46.67 \%$ of participants lived at poverty level (annual average income below $\$ 4000)$. There was a high prevalence of abnormal weight (35\% underweight, $21.67 \%$ overweight, and $8.33 \%$ obesity). Moreover, with respect to participants' social situation, 30\% were unmarried, 25\% were living alone, 40\% seldom or never had contact with family, and $25 \%$ seldom or never had contact with friends.

\subsection{Performance evaluation of the WSMD}

The mean and standard deviation of vital sign data in the participant sample, measured using the WSMD and traditional instruments, are provided in Table 2. Results of independent sample tests are shown in Table 3. Differences in vital sign data measurement between the WSMD and traditional instrument groups showed no statistical significance $(\mathrm{p}>0.05)$. Therefore, we believe that the WSMD is an effective method for monitoring vital signs. A total of 10 repetitive measurements were taken using the WSMD, under the same circumstances. 
The relative standard deviation of this method was $0.38 \%$, which indicates good repeatability.

Abbreviations: Temp, temperature; SBP, systolic blood pressure; DBP, diastolic blood pressure; $\mathrm{PR}$, pulse rate; $\mathrm{SpO}$, blood oxygen saturation; A, WSMD measurement; B, traditional instrument measurement.

Table 1. Characteristics of the study samples

\begin{tabular}{cccc}
\hline Factors & Group & $\begin{array}{c}\text { Participants } \\
(\mathrm{n}=180)\end{array}$ & $\begin{array}{c}\text { Percent } \\
(\%)\end{array}$ \\
\hline Sex & Female & 96 & 53.33 \\
Male & $18-29$ & 84 & 46.67 \\
& $30-39$ & 63 & 35.00 \\
& $40-49$ & 54 & 30.00 \\
& $50-59$ & 18 & 10.00 \\
& $\geqslant 60$ & 27 & 15.00 \\
& No formal education & 18 & 10.00 \\
& Junior high school & 30 & 10.00 \\
& Senior high school & 42 & 23.33 \\
& Junior college & 45 & 25.00 \\
& University & 45 & 25.00 \\
& $<1000 \$$ & 33 & 20.00 \\
Annual Average & $1000-4000 \$$ & 48 & 26.67 \\
& $4000-10000 \$$ & 45 & 25.00 \\
& $10000-15000 \$$ & 33 & 18.33 \\
& $>15000 \$$ & 18 & 10.00 \\
& & &
\end{tabular}

Body Mass Index

$$
\begin{aligned}
& \text { Underweight }\left(<18.5 \mathrm{~kg} / \mathrm{m}^{2}\right) \\
& \text { Normal weight }(18.5-24.9 \\
& \left.\mathrm{kg} / \mathrm{m}^{2}\right) \\
& \text { Overweight }(25.0-29.9 \\
& \left.\mathrm{kg} / \mathrm{m}^{2}\right) \\
& \text { Obesity }\left(\geqslant 30.0 \mathrm{~kg} / \mathrm{m}^{2}\right) \\
& \text { Unmarried } \\
& \text { Married } \\
& \text { Alone }
\end{aligned}
$$

Marital Status

Living Status

Family Contact

Friend Contact

Not Alone

Every week

Every month

Seldom or never

Every week

Every month

Seldom or never

Table 2. Group statistics of data measured by the WSMD and traditional instruments

\begin{tabular}{cccccc}
\hline & Group & $\mathrm{N}$ & Mean & Std. Deviation & Std. Error Mean \\
\hline \multirow{2}{*}{ Temp } & A & 15 & 36.5933 & 0.12228 & 0.03157 \\
& B & 15 & 36.5933 & 0.10998 & 0.02840 \\
SBP & A & 15 & 123.3333 & 8.05930 & 2.08090 \\
& B & 15 & 122.3333 & 7.98809 & 2.06252 \\
DBP & A & 15 & 85.0000 & 2.90320 & 0.74960 \\
& B & 15 & 85.3333 & 2.96808 & 0.76636 \\
$\mathrm{PR}$ & A & 15 & 76.0000 & 1.41421 & 0.36515 \\
& B & 15 & 75.6000 & 1.12122 & 0.28950 \\
$\mathrm{SpO}_{2}$ & A & 15 & 98.0667 & 0.70373 & 0.18170 \\
& B & 15 & 97.8000 & 0.41404 & 0.10690 \\
\hline
\end{tabular}

Table 3. Independent sample testing of data measured by the WSMD and traditional instruments

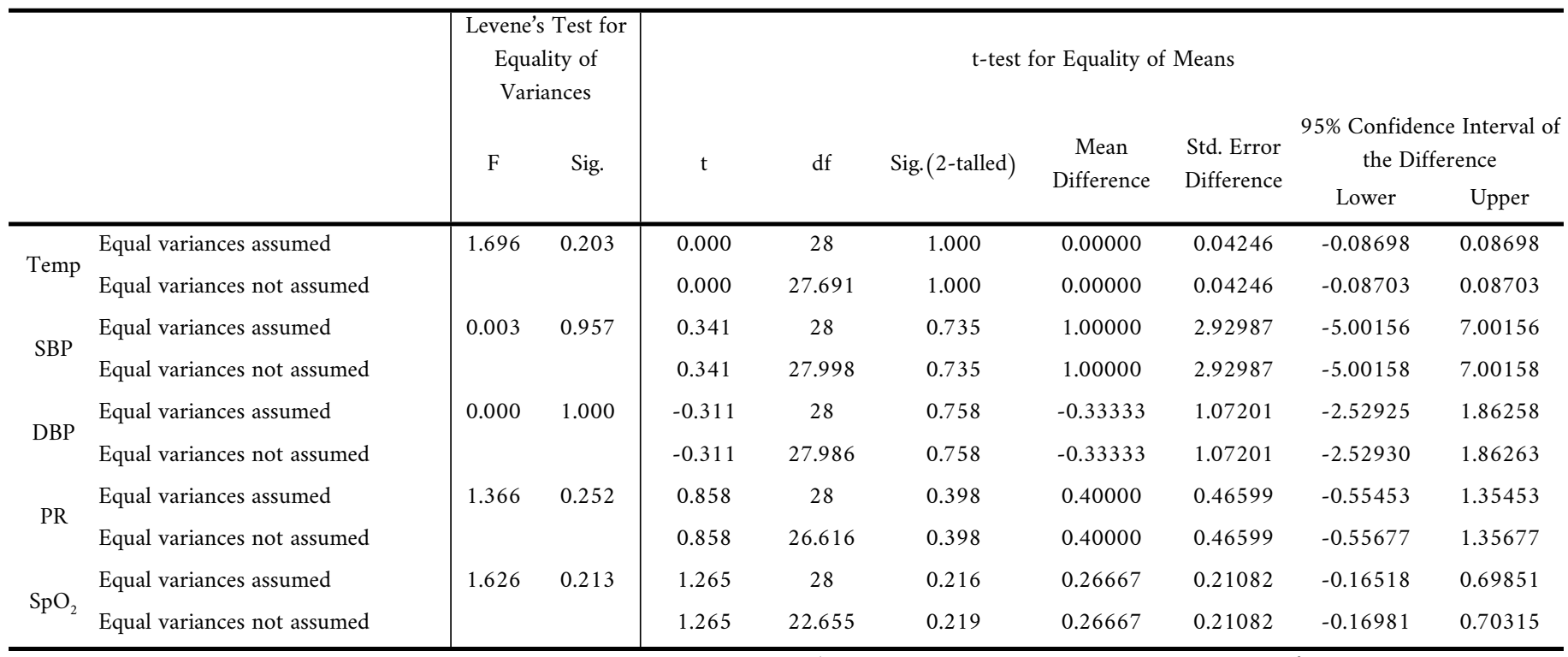

\subsection{Evaluation of effectiveness of the SMM}

Descriptive statistics are presented in Table 4. The rankings shown in Table 5 indicate that for Q1 after intervention, the frequency of recording dietary information increased among 153 participants and remained unchanged for 27 participants. With respect to $\mathrm{Q} 2$, choosing their diet according to health status was improved in 141 participants and was the same in the remaining participants. For Q3 after intervention, 159 raised their levels of attention paid to physical changes after eating; 21 participants remained unchanged. The test statistics presented in Table 6 show that the differences in Q1, Q2 and Q3 between the two groups were significant $(\mathrm{p}<0.05)$. After using the SMM, participants' attention to vital signs and diet improved significantly compared with before the intervention. Thus, this SMM can help bring about effective improvement in 
personal health monitoring and diet optimization, and therefore, improved HRQoL.

Table 4. Descriptive statistics before and after intervention

\begin{tabular}{lcccccc}
\hline & Group & $\mathrm{N}$ & Mean & Std. Deviation & Minimum & Maximum \\
\hline Q1 & Before & 180 & 1.6500 & 0.70493 & 1.00 & 4.00 \\
& After & 180 & 3.1167 & 1.05319 & 2.00 & 5.00 \\
Q2 & Before & 180 & 2.6667 & 0.74744 & 1.00 & 4.00 \\
& After & 180 & 3.6500 & 0.72832 & 2.00 & 5.00 \\
Q3 & Before & 180 & 1.5667 & 0.64384 & 1.00 & 3.00 \\
& After & 180 & 2.9000 & 0.99776 & 2.00 & 5.00 \\
\hline
\end{tabular}

\section{Discussion}

Providing medical care using sensors and mobile technology for the self-management of health and disease is a growing trend toward improving HRQoL [36-38]. The personal health record (PHR) has been shown to have a great influence on personal health care $[39,40-42]$. Here, we proposed a method, the SMM, for monitoring vital signs and dietary nutritional data as part of a PHR. The SMM is based on a WBAN and mobile technology and comprises both WSMD and HSMS. Data analysis in the SMM contains two levels: unitary attribute data analysis and synthetic data analysis. In the first level, only differences between collected user data and reference data are examined. In this way, the most serious anomalies can be quickly discovered. The second level refers to investigation of the relationships between physical changes and diet, to determine background factors related to health. Such analysis might prevent health crises before they emerge. To conduct these two levels of analysis, three expert knowledge bases were established, denominated the RDB, FNDB and DRDB. Normal reference ranges of vital signs were stored in the $\mathrm{RDB}$ for data comparison. The FNDB contained a catalogue of nutrients and calories for each unit of food. In addition, personalized recommendations and restrictions were recorded in the DRDB, to evaluate whether the user's choice of diet was suitable.

Table 5. Ranking

\begin{tabular}{|c|c|c|c|c|}
\hline & & $\mathrm{N}$ & Mean Rank & Sum of Ranks \\
\hline \multirow[t]{4}{*}{ Q1: After-Before } & Negative Ranks & $0^{\mathrm{a}}$ & 0.00 & 0.00 \\
\hline & Positive Ranks & $153^{\mathrm{b}}$ & 77.00 & 11781.00 \\
\hline & Ties & $27^{\mathrm{c}}$ & & \\
\hline & Total & 180 & & \\
\hline \multirow[t]{4}{*}{ Q2: After-Before } & Negative Ranks & $0^{\mathrm{a}}$ & 0.00 & 0.00 \\
\hline & Positive Ranks & $141^{\mathrm{b}}$ & 71.00 & 10011.00 \\
\hline & Ties & $39^{\mathrm{c}}$ & & \\
\hline & Total & 180 & & \\
\hline \multirow[t]{4}{*}{ Q3: After-Before } & Negative Ranks & $0^{\mathrm{a}}$ & 0.00 & 0.00 \\
\hline & Positive Ranks & $159^{\mathrm{b}}$ & 80.00 & 12720.00 \\
\hline & Ties & $21^{\mathrm{c}}$ & & \\
\hline & Total & 180 & & \\
\hline
\end{tabular}

a: After Intervention < Before Intervention; b: After Intervention > Before Intervention; c: After Intervention = Before Intervention.

Table 6. Test statistics ${ }^{\mathrm{b}}$

\begin{tabular}{lrr}
\hline & Q1 & Q2 \\
& After -Before & After -Before \\
\hline Asymp.Sig.(2-tailed) & $-11.027^{\mathrm{a}}$ & $-10.994^{\mathrm{a}}$ \\
After -Before & $0.11 .398^{\mathrm{a}}$ & 0.000 \\
\hline
\end{tabular}

a: Based on negative ranking; b: Wilcoxon signed-rank test.

This study demonstrated that there was no statistical significance between the SMM developed here and traditional instruments for acquiring vital sign data, and indicated that the SMM has good repeatability. These findings suggest that the SMM has a positive promoting effect on improvement in health habits, health monitoring, and disease prevention. In our study, $85 \%$ of participants improved their habits of dietary recordkeeping after the intervention, with nearly half reporting never having recorded their dietary intake before intervention; in fact, $35 \%$ of participants demonstrated two levels of improvement with respect to keeping track of their diet. More than half of participants reported usually or always choosing their food and cooking methods with their health in mind after the intervention, as compared with $13.33 \%$ before intervention. Several participants became more aware of physical changes after eating, with a substantial increase from $8.33 \%$ to $56.67 \%$ after intervention. These statistics indicate that the SMM can contribute to updating concepts and behaviors regarding personal health, and improve awareness about physical conditions and a balanced diet. The impacts on HRQoL are beneficial for people at all levels of physical health. 
The SMM developed in this study relied on daily measurement and recording. The high level of participation required a certain level of knowledge and skills. Therefore, people who did not use a smartphone or those who were illiterate were not taken into account. In this study, we gathered information of Temp, BP, PR, and SpO2 using the SMM. In future research, sensors for additional physical parameters should be added. On the premise of sharing data voluntarily, a foundation of health care big data can be established. The SMM can also provide reliable data for use in research on disease and the dietary situations of an entire country or region, and can serve as a reference for life quality improvement.

\section{Conclusion}

Vital signs reflect the physical state of humans, and diet is an intrinsic factor affecting human health. Owing to the importance of consistent monitoring of these elements, we proposed a self-monitoring method comprising a WSMD element and HSMS. Our results showed that this method was accurate, reproducible, and could effectively improve HRQoL. The SMM can be used for the acquisition, management, monitoring, and analysis of health-related data. This work lays a foundation for establishing intelligent mobile health services and health big data analysis.

\section{Acknowledgement}

This work was supported by the Research and Development Program of Shandong Province (2019GSF111035).

\section{References}

[1] Evans, D., Hodgkinson, B., \& Berry, J. (2001). Vital signs in hospital patients a systematic review. International Journal of Nursing Studies, 38(6), 643-650.

[2] Dall'Ora C., Griffiths P., Hope J., Briggs J., Jeremy J., Gerry S., \& Redfern O.C. (2021). How long do nursing staff take to measure and record patients' vital signs observations in hospital? a time-and-motion study. International Journal of Nursing Studies. 118(103921), 1-7.

[3] Harries, A.D., Zachariah, R., Kapur, A., Jahn, A., \& Enarson, D.A. (2009). The vital signs of chronic disease management. TRANSACTIONS OF THE ROYAL SOCIETY OF TROPICAL MEDICINE AND HYGIENE, 103(6), 537-540.

[4] Kumar, V. S. , \& Krishnamoorthi, C. . (2021). Development of electrical transduction based wearable tactile sensors for human vital signs monitor: fundamentals, methodologies and applications. Sensors and Actuators A Physical, 321 (1), 112582.

[5] Leonard, M.M., \& Kyriacos, U. (2015). Student nurses' recognition of early signs of abnormal vital sign recordings. Nurse Education Today, 35(9), 11-18.

[6] Mok, W.Q., Wang, W.R., \& Liaw, S.Y. (2015). Vital signs monitoring to detect patient deterioration: An integrative literature review. INTERNATIONAL JOURNAL OF NURSING PRACTICE, 21 (2), 91-98.
[7] Lou, D.D., Chen, X.X., Zhao, Z., Xuan, Y.D., Xu, Z.H., Jin, H., Guo, X.Z., \& Fang, Z. (2013). A Wireless Health Monitoring System based on Android Operating System. 2013 INTERNATIONAL CONFERENCE ON ELECTRONIC ENGINEERING AND COMPUTER SCIENCE (EECS 2013). 4, 208-215.

[8] Kassianos, A.P., Raats, M.M., Gage, H., \& Peacock, M. (2015). Quality of life and dietary changes among cancer patients: a systematic review. QUALITY OF LIFE RESEARCH, 24(3), 705-719.

[9] Reedy, J., Krebs-Smith, S.M., Miller, P.E., Liese, A.D., Kahle, L.L., Park, Y., \& Subar, A.F. (2014). Higher Diet Quality Is Associated with Decreased Risk of All-Cause, Cardiovascular Disease, and Cancer Mortality among Older Adults. JOURNAL OF NUTRITION, 144(6), 881-889.

[10] van't Riet, J., Sijtsema, S.J., Dagevos, H., \& De Bruijn, G.J. (2011). The importance of habits in eating behaviour. An overview and recommendations for future research. Appetite, 57(3), 585-596.

[11] Fernandez, R., Rolley, J.X., Rajaratnam, R., Everett, B., \& Davidson, P.M. (2015). Reducing the risk of heart disease among Indian Australians: knowledge, attitudes, and beliefs regarding food practices - a focus group study. FOOD ef NUTRITION RESEARCH, doi: 10.3402/fnr.v59.25770.

[12] Rose, D., Heller, M. C., \& Roberto, C. A.. (2019). Position of the society for nutrition education and behavior: the importance of including environmental sustainability in dietary guidance. Journal of Nutrition Education and Behavior, 51(1), 3-15.e1.

[13] Yilmaz, T., Foster, R., \& Hao, Y. (2010). Detecting Vital Signs with Wearable Wireless Sensors. SENSORS, 10(12), 10837-10862.

[14] Burnier, M., Wuerzner, G., \& Bochud, M. (2015). Salt, blood pressure and cardiovascular risk: what is the most adequate preventive strategy? A Swiss perspective. FRONTIERS IN PHYSIOLOGY, 6, 1-9.

[15] Farquhar, W.B., Edwards, D.G., Jurkovitz, C.T., \& Weintraub, W.S. (2015). Dietary Sodium and Health More Than Just Blood Pressure. JOURNAL OF THE AMERICAN COLLEGE OF CARDIOLOGY, 65(10), 1042-1050.

[16] Feng Z., Glinskaya E., Chen H., Gong S., Qiu Y., Xu J., \& Yip W. (2020). Long-term care system for older adults in china: policy landscape, challenges, and future prospects. The Lancet, 396(10259):1362-1372.

[17] Giger, J.T., Pope, N.D., Vogt, H.B., Gutierrez, C., Newland, L.A., Lemke, J., \& Lawler, M.J. (2015). Remote patient monitoring acceptance trends among older adults residing in a frontier state. COMPUTERS IN HUMAN BEHAVIOR, 44, 174-182.

[18] Li, C.T., Chen, F.M., Jin, J.X., Lv, H., Li, S., Lu, G.H., \& Wang, J.Q. (2015). A Method for Remotely Sensing Vital Signs of Human Subjects Outdoors. SENSORS, 15(7), 14830-14844.

[19] Steinhubl, S.R., Marriott, M.P., \& Wegerich, S.W. (2015). Remote Sensing of Vital Signs: A Wearable, Wireless "BandAid" Sensor With Personalized Analytics for Improved Ebola Patient Care and Worker Safety. Global health, science and practice, 3(3), 516-519.

[20] Yang, Z.B., Kankanhalli, A., Ng, B.Y., \& Lim, J.T.Y. (2015). Examining the pre-adoption stages of healthcare IT: A case 
study of vital signs monitoring systems. Information eb Management, 52 (4), 454-467.

[21] Ishijima, M. (2007). Unobtrusive approaches to monitoring vital signs at home. MEDICAL \& BIOLOGICAL ENGINEERING \& COMPUTING, 45(11), 1137-1141.

[22] Hung, L. P. , \& Lin, C. C. . (2019). A multiple warning and smart monitoring system using wearable devices for home care. International Journal of Human-Computer Studies, 136, 102381.

[23] Pecina, J.L., Hanson, G.J., Van Houten, H., \& Takahashi, P.Y. (2013). Impact of telemonitoring on older adults healthrelated quality of life: the Tele-ERA study. QUALITY OF LIFE RESEARCH, 2013, 22(9), 2315-2321.

[24] Sun, N., \& Rau, P.L.P. (2015). The acceptance of personal health devices among patients with chronic conditions. International Journal of Medical Informatics, 84(4), 288-297.

[25] Medina, F.X. (2009). Mediterranean diet, culture and heritage: challenges for a new conception. Public health nutrition, 12(9), 1618-1620.

[26] Jang, S., \& Kim, D. (2015). Enhancing ethnic food acceptance and reducing perceived risk: The effects of personality traits, cultural familiarity, and menu framing. INTERNATIONAL JOURNAL OF HOSPITALITY MANAGEMENT, 47, 85-95.

[27] Ohtomo, S. (2013). Effects of habit on intentional and reactive motivations for unhealthy eating. Appetite, 68, 69-75.

[28] Sen, S., Subbaraju, V., Misra, A., Balan, R.K. \& Lee, Y. (2015). The case for smartwatch-based diet monitoring. 2015 IEEE International Conference on Pervasive Computing and Communication Workshops (PerCom Workshops), 585-590.

[29] Amft, O., \& Troster, G. (2008). Recognition of dietary activity events using on-body sensors. ARTIFICIAL INTELLIGENCE IN MEDICINE, 42(2), 121-136.

[30] Dong, Y.J., Hoover, A., Scisco, J., \& Muth, E. (2012). A New Method for Measuring Meal Intake in Humans via Automated Wrist Motion Tracking. APPLIED PSYCHOPHYSIOLOGY AND BIOFEEDBACK, 37(3), 205215.

[31] Dong, B., \& Biswas, S. (2013). Wearable Diet Monitoring Through Breathing Signal Analysis. IEEE Engineering in Medicine and Biology Society Conference Proceedings, 11861189.

[32] Ullah, S., Khan, P., Ullah, N., Saleem, S., Higgins, H., \& Kwak K.S. (2009). A Review of Wireless Body Area Networks for Medical Applications. International Journal of Communications, Networks and System Sciences, 2(8), 797-803.

[33] Hajar, M. S. , Al-Kadri, M. O. , \& Kalutarage, H. K. . (2021). A survey on wireless body area networks: architecture, security challenges and research opportunities. Computers es Security. 104, 1-27.

[34] Laine, T.H., Lee C., \& Suk H. (2014). Mobile Gateway for Ubiquitous Health Care System Using ZigBee and Bluetooth. 2014 Eighth International Conference on Innovative Mobile and Internet Services in Ubiquitous Computing (IMIS). Proceedings, 139-145.

[35] Challoo, R., Oladeinde, A., Yilmazer, N., Ozcelik, S., \& Challoo, L. (2012). An Overview and Assessment of Wireless Technologies and Co-existence of ZigBee, Bluetooth and WiFi Devices. Procedia Computer Science,12, 386-391.
[36] Al Dandah, M., Du Lou, A.D., \& Meadel, C. (2015). Mobile health and maternal care: A winning combination for healthcare in the developing world? HEALTH POLICY AND TECHNOLOGY, 4(3), 225-231.

[37] Royston, G., Hagar, C., Long, L.A., McMahon, D., PakenhamWalsh, N., \& Wadhwani, N. (2015). Mobile health-care information for all: a global challenge. The Lancet Global Health, 3(7), 356-357.

[38] Bradway, M., Arsand, E., \& Grottland, A. (2015). Mobile Health: empowering patients and driving change. Trends in Endocrinology and Metabolism, 2015, 26(3), 114-117.

[39] Baudendistel, I., Winkler, E., Kamradt, M., Brophy, S., Langst, G., Eckrich, F., Heinze, O ., Bergh, B., Szecsenyi, J., \& Ose, D. (2015). The patients' active role in managing a personal electronic health record: a qualitative analysis. SUPPORTIVE CARE IN CANCER, 23(9), 2613-2621.

[40] Gee, P.M., Paterniti, D.A., Ward, D., \& Soederberg Miller, L.M. (2015). e-Patients Perceptions of Using Personal Health Records for Self-management Support of Chronic Illness. Computers, informatics, nursing: CIN, 33(6), 229-237.

[41] Gartrell, K., Trinkoff, A.M., Storr, C., \& Wilson, M.L. (2015). Electronic Personal Health Record Use Among Nurses in the Nursing Informatics Community. Computers, informatics, nursing : CIN, 33(7), 306-314.

[42] Muzny, M., Henriksen, A., Giordanengo, A., Muzik, J., Grottland, A., Blixgard, H., Hartvigsen G. \& Årsand E.. (2020). Wearable sensors with possibilities for data exchange: analyzing status and needs of different actors in mobile health monitoring systems. International journal of medical informatics, 133, 104017.1-104017.8.

\section{BIOGRAPHIES}

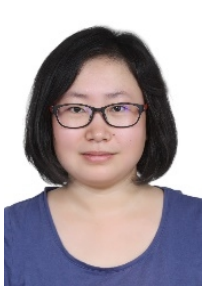

Lina Yu is currently an assistant research fellow with the Institute of Semiconductors, Chinese Academy of Sciences. Her researches focus on machine learning, deep modeling, and intelligent system.

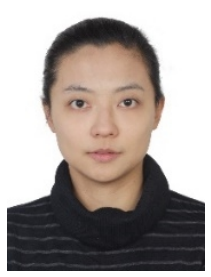

Sha Tao is currently an associate professor of College of Information and Electrical Engineering, China Agricultural University. Her research mainly focuses on processing and storage of agriculture products technologies.

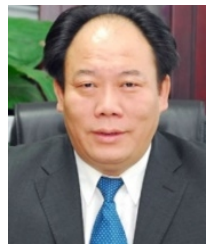

Wanlin Gao is a professor of College of Information and Electrical Engineering, China Agricultural University. His research interests include the informationization of new rural areas, intelligence agriculture, and the service for rural comprehensive information. 
Limin Yu PhD candidate, College of Information Science and Engineering, China Agricultural University. Her research interest: Agricultural informationization and application. 